\title{
Design of Superionic Polymer Electrolytes
}

\author{
Yangyang Wang ${ }^{1}$ and Alexei P. Sokolov ${ }^{1,2}$
}

\begin{abstract}
Despite potential significant advantages of polymer based batteries, the poor ionic conductivity of dry polymer electrolytes at ambient and low temperatures has limited their application. This review describes the approach for improving conductivity by decoupling ionic transport from polymer segmental relaxation. It is emphasized that the decoupling approach is the key for design of superionic polymer electrolytes.

Addresses

${ }^{1}$ Department of Chemistry, University of Tennessee, Knoxville, Tennessee 37996, USA

${ }^{2}$ Chemical Sciences Division, Oak Ridge National Laboratory, Oak Ridge, Tennessee 37831, USA

Corresponding authors: Wang, Yangyang (yywang@utk.edu) and Sokolov, Alexei P. (sokolov@utk.edu)
\end{abstract}

\section{Introduction}

Polymer electrolytes are an important class of solid-state ionic conductors that hold promises for a wide variety of applications in electrochemical devices $\left[1-5,6 * *, 7,8^{*}\right]$. Replacing organic liquid electrolytes with polymer electrolytes would significantly improve safety, reduce weight, and increase energy density for batteries. Nevertheless, the poor ionic conductivity of dry polymer electrolytes at ambient and low temperatures has remained a major obstacle for full exploitation of these materials in vehicle propulsion and other high-power applications. According to the classical theory, ionic transport in polymers is closely coupled to segmental (structural) dynamics $\left[1,2,6^{* *}, 9,10\right]$ [Fig. 1(a)]. Based on this understanding the main direction of research during the past several decades has been focused on developing polymers with low glass transition temperatures, where fast segmental dynamics would lead to high conductivity. However, it has become increasingly clear that this approach might not be the correct strategy to achieve the desired conductivity level of $10^{-3} \mathrm{~S} / \mathrm{cm}$ at ambient temperature $\left[11^{* *}, 12,13 * *, 14 *\right]$. It is also possible to speed up the segmental dynamics by plasticizing polymers with some small 
molecules. However, this will necessarily compromise some advantages that polymer electrolytes promise to offer.

As a result, a radically different strategy, the decoupling approach, has been proposed $\left[5,11^{* *}, 12,13^{* *}, 14^{*}, 15^{*}, 16,17^{*}, 18,19,20^{*}, 21-26,29,30^{*}\right]$. The main idea is to decouple the ionic transport from the structural dynamics of the polymer matrix [Fig. 1(b)]. We emphasize that superionic glasses and crystals [31**,32-37], another promising candidate for battery electrolytes, exhibit exceptionally high solid-state conductivity due to their "decoupled" ion conduction mechanism. In that case, the rate of ion jumps is significantly faster than the rate of structural relaxation. However, their intrinsically poor mechanical property (brittleness) makes them unsuitable for many applications. Therefore, realizing superionic conduction in polymers has become one of the "holy grails" in electrolyte science.

Here we present a brief overview of the efforts to achieve superionic conductivity in polymer electrolytes during the last two decades or so, and stresses the new insights from the recent analyses of the relation between ionic transport and structural dynamics in polymers.

\section{Relation between ionic transport and structural relaxation}

Study of the relation between ionic transport and relaxation phenomenon is a central task in electrolyte science and engineering. To understand and thereby improve the ionic conductivity of polymer electrolytes, one needs to analyze the relation between ionic transport and polymer segmental dynamics. However, after decades of research, the mechanism of ion conduction in polymers is still not fully understood. Moreover, the highest conductivities achieved in solventfree polymer electrolytes at ambient temperature are still roughly two or three orders of magnitude lower than those of organic liquid electrolytes and conducting ceramics. This difficulty of finding a polymer with sufficient ionic conductivity has raised a fundamental question: are polymer electrolytes intrinsically inferior to other electrolytes in terms of their charge transport capability? The recent analyses $\left[11^{* *}, 14^{*}, 38\right]$ of the relation between ionic transport and structural relaxation in polymeric and molecular electrolytes have provided an encouraging and yet surprising answer to this question.

According to the classical theory, the ionic conductivity of an electrolyte solution is determined by the number density $(n)$, ionic mobility $\left(\mu_{\mathrm{q}}\right)$, and charge $(q)$ of all its ionic constituents $(i)$ :

$$
\sigma=\sum_{i} n_{i} \mu_{q, i} q_{i}
$$


Among these three factors, the ionic mobility, which can vary many orders of magnitude upon change of temperature, often plays a decisive role. The ionic mobility is related to the diffusivity (D) of the ions through the Einstein relation:

$$
\mu_{q}=\frac{D q}{k_{B} T} .
$$

Simple consideration of the Stokes law leads to the prediction that the ionic mobility is inversely proportional to the solution viscosity $(\eta)$ and proportional to the solvent structural relaxation rate $\left(\omega_{\mathrm{S}}\right)$ :

$$
\mu_{q} \propto \omega_{\mathrm{s}}
$$

It has been shown that the Stokes law does not work for many ions in aqueous solutions. Hydrodynamic and microscopic theories have been developed to take into account the effects of dielectric friction [39-41] and ultrafast solvent dynamics [42]. Nevertheless, the proportionality between mobility and structural relaxation rate is still expected to hold to the first approximation. This is supported by temperature-dependent measurements of $\mu_{\mathrm{q}}$ and $\omega_{\mathrm{s}}$ of molecular electrolyte solutions. The applicability of Eq. 3 to polymer electrolytes is the central point of this review. Experimentally, it is possible to determine both $\mu_{\mathrm{q}}$ and $\omega_{\mathrm{s}}$ from impedance/dielectric spectroscopy measurement. The ionic mobility can be evaluated from the measured dc conductivity $\sigma$ using Eq. 1, if the density of "free" ions $n_{i}$ is known or assumed.
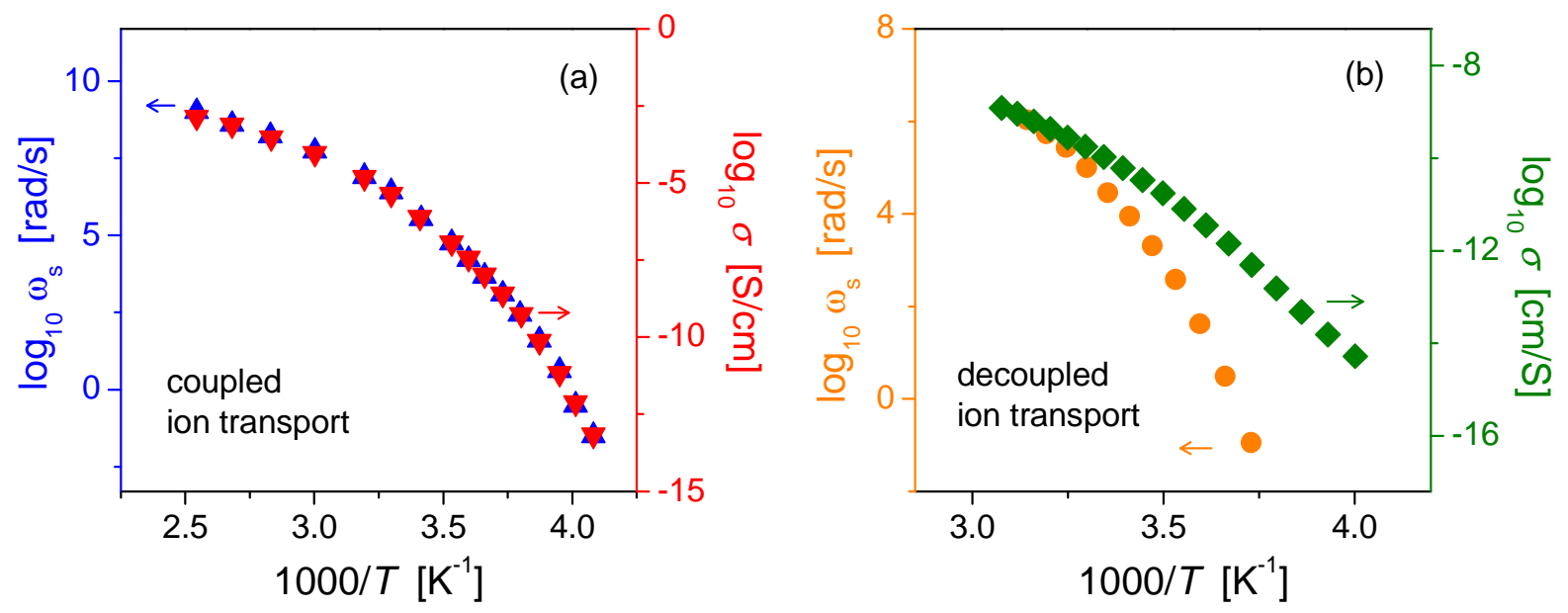

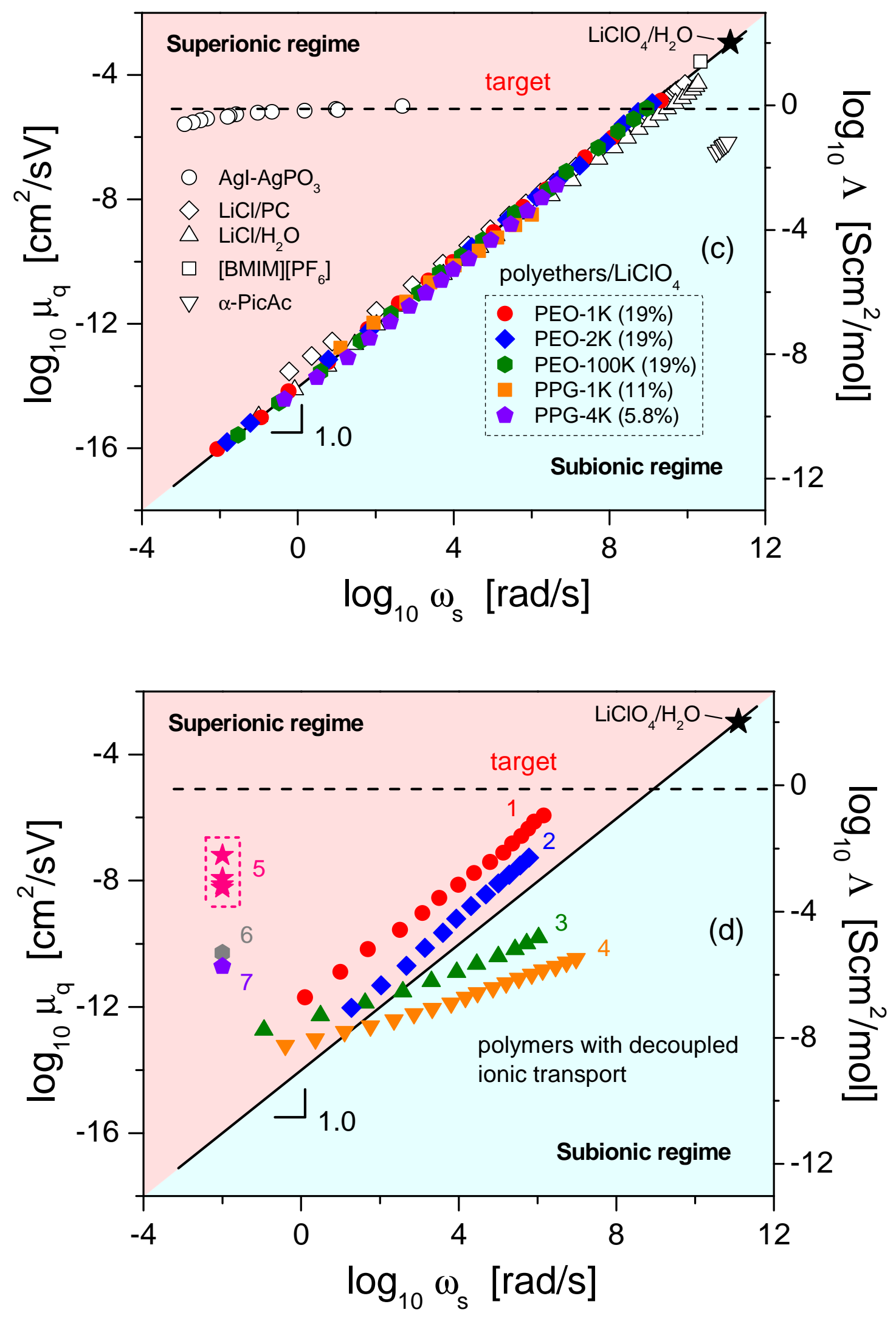
Figure 1. Temperature dependence of segmental relaxation frequency $\left(\omega_{\mathrm{s}}\right)$ and dc conductivity $(\sigma)$ for (a) coupled [43] and (b) decoupled polymer electrolytes [13**]. (c) Relation between $\mu_{\mathrm{q}}$ and $\omega_{\mathrm{s}}, \Lambda$ and $\omega_{\mathrm{s}}$, for polyethers [14*,43] and the non-polymeric ionic conductors ([11**] and refs. therein). The black line with the slope one is the "ideal" Walden line, representing the case where the conductivity is strongly coupled to structural relaxation. The horizontal dashed line indicates the target $\mu_{\mathrm{q}}$ and $\Lambda\left[11^{* *}, 14^{*}\right]$. (d) Relation between $\mu_{\mathrm{q}}$ and $\omega_{\mathrm{s}}, \Lambda$ and $\omega_{\mathrm{s}}$, for polymers with decoupled ionic conductivity. Samples 1-7 are: 1 - PVC-PPEGMEMA/LiTFSI [11**], 2 PVEC-PVAc/LiTFSI [11**], 3 - PPEOSt-PSt/LiClO 4 [11**,13**], 4 - PMOEOMSt/LiClO 4 [11**,13**], 5- liquid-crystalline polymers with $\mathrm{LiClO}_{4}$ [19], 6 - 0.8(0.5 LiPUS-AlCl 3$)$ $0.2 \mathrm{LiAlCl}_{4}$ [23], and 7 - 90\%(50LiI-30LiOAc-20LiClO 4$)+10 \% \mathrm{PPO}\left[15^{*}\right]$.

A very useful classification of ionic conductors can be made by analyzing the relation between ionic transport and structural relaxation, i.e., performing the so-called (modified) Walden plot analysis [11**,14*,44] [Fig.1 (c), (d)]. To quantify the "intrinsic" conducting capability of ionic materials, their molar conductivities ( $\Lambda=\sigma / n_{\text {tot }}$, with $n_{\text {tot }}$ being the total number density of ions) or ionic mobilities $\left(\mu_{\mathrm{q}}\right)$ can be presented as a function of their structural relaxation frequencies $\left(\omega_{\mathrm{s}}\right)$. A straight line of slope one, passing through a reference dilute salt aqueous solution such as $\mathrm{KCl} / \mathrm{H}_{2} \mathrm{O}$ and $\mathrm{LiClO}_{4} / \mathrm{H}_{2} \mathrm{O}$, is often called the "ideal" Walden line. It divides the plane into superionic (above) and subionic (below) regimes. "Superior" ionic conductors are those that exhibit ionic mobility (or molar conductivity) much higher than the ideal line. Moreover, the degree of decoupling between conductivity and segmental dynamics can be quantified by using the exponent $\alpha$ from the fractional Stokes-Einstein relation [13**]: $\mu_{\mathrm{q}}=\omega_{\mathrm{s}}^{\alpha}$ or $\Lambda=\omega_{\mathrm{s}}^{\alpha}$ : the smaller the $\alpha$, the larger the degree of decoupling. $\alpha=1$ corresponds to the case of complete coupling, while $\alpha \sim 0$ (e.g. $\mathrm{AgI}-\mathrm{AgPO}_{3}$ glass, Fig. 1b) implies completely decoupled ion motions. A few examples of this classification of liquid small-molecule electrolytes and ceramic conductors are given in Fig. 1(c). Three of the small-molecule electrolytes, $\mathrm{LiCl} / \mathrm{propylene}$ carbonate (PC), $\mathrm{LiCl} / \mathrm{H}_{2} \mathrm{O}$, and 1-Butyl-3-methylimidazolium hexafluorophosphate ([BMIM] $\left[\mathrm{PF}_{6}\right]$ ) closely follow the ideal line, indicating strong coupling between ionic transport and structural relaxation, as well as good ion solvation. The inorganic salt, $\mathrm{AgI}-\mathrm{AgPO}_{3}$, stays above the ideal line, exhibiting superionic behavior. The protic ionic conductor, $\alpha$-picolinium acetate $(\alpha-\mathrm{PicAc})$, appears in the subionic regime because of poor ion dissociation. 
It should be noted that the original Walden plot analysis was performed in terms of fluidity $(1 / \eta)$, instead of structural relaxation frequency $\left(\omega_{\mathrm{s}}\right)$. This did not allow the Walden plot analysis to be properly applied to polymer electrolytes, because the ionic transport in polymers is controlled by microscopic rather than macroscopic viscosity [11**]. However, it was demonstrated recently that by using structural relaxation frequency, the Walden plot analysis could be successfully extended to polymer electrolytes, yielding new insights about the ionic transport mechanism in polymers $\left[11^{* *}, 14^{*}, 38\right]$. The results of these analyses are summarized below.

In the case of polyethers such as PEO and PPG, it is well known that their conductivity is closely coupled to their segmental relaxation. The Walden plot analysis further reveals $\left[11^{* *}, 14^{*}, 38\right]$ that the ionic conductivity in polyethers falls surprisingly close to the "ideal" Walden line of aqueous solutions [Fig. 1(c)]. This suggests that the ionic transport (friction) mechanism in polyethers is very similar to those of small-molecule liquid electrolytes. It follows that the relatively low conductivity in polyethers compared with liquid organic electrolytes is mainly a result of their sluggish segmental dynamics. For polyethers to reach the target conductivity of $10^{-}$ ${ }^{3} \mathrm{~S} / \mathrm{cm}$, the segmental relaxation frequency has to be $\sim 10^{8} \mathrm{rad} / \mathrm{s}$ or higher, even assuming $50 \mathrm{wt} \%$ loading of lithium bis(trifluoromethane) sulfonimide (LiTFSI) and its complete dissociation [14*]. In the case of PEO, this is only possible at $\sim 80^{\circ} \mathrm{C}$ and above. This analysis consolidates the long speculation about the limited performance of polyether-based electrolytes [21]. In other words, it seems impossible for polyether-based electrolyte to reach the required conductivity at ambient and low temperatures, unless the polymer is plasticized.

In contrast to polyethers, the ionic transport in other polymers can be strongly decoupled from their segmental dynamics. In general, two types of decoupling behavior have been observed [11**] [Fig. 1(d)]. In materials such as poly[poly(ethylene oxide) styrene]-polystyrene (PPEOStPSt) and poly[4-(2-methoxyethoxy)methyl styrene] (PMOEOMSt), ionic conductivity and polymer segmental relaxation exhibit very different temperature dependence, giving rise to prominent fractional Stokes-Einstein behavior. Although these samples fall into the subionic regime at high temperatures due to low free ion concentration (poor ion solvation), the decoupling between conductivity and segmental relaxation brings them in the superionic regime at low temperatures. A different kind of decoupling behavior is observed in polymers such as poly(vinyl carbonate)-co-poly[poly(ethylene glycol methyl ether methacrylate)] (PVCPPEGMEMA) and poly(vinyl ethylene carbonate)-co-poly(vinyl acetate) (PVEC-PVAc), where 


\section{Different approaches to decoupling}

The concept of decoupling of ion conductivity from segmental dynamics has been around for a long time. Already in 1993, Angell and coworkers [15*], using PPG-based electrolytes as an example, proposed the concept of polymer-in-salt electrolytes. In such materials, inorganic salts are the major component and a small amount of polymers serve as a binder or plasticizer. The main idea of the polymer-in-salt electrolytes strategy is to keep the polymer concentration required for good mechanical properties to the minimum, in the hope that the ion conduction mechanism in such highly concentrated electrolytes will approach those of inorganic salts, where relative high and even superionic conductivity is commonly observed.

The pioneering work of Angell and coworkers opened the venue of design of superionic polymers. Ferry, Forsyth, MacFarlane, and coworkers [18,22,45] later extended this polymer-insalt electrolytes approach to a series of other solid polymers, including poly(acrylonitrile) (PAN), poly(1-vinyl pyrrolidone) (PVP), and poly( $N, N$-dimethyl acrylamide) (PDMAA), and conductivities greater than or equal to $10^{-8} \mathrm{~S} / \mathrm{cm}$ at the glass transition temperature $\left(T_{\mathrm{g}}\right)$ were observed $\left(\sigma \sim 10^{-15} \mathrm{~S} / \mathrm{cm}\right.$ is expected at $T_{\mathrm{g}}$ in the case of coupled ionic conductivity, see e.g. Fig.1a).

Around the same period, another approach for decoupling conductivity and segmental relaxation in the traditional salt-in-polymer electrolytes emerged from the work of several different groups. The key idea is to frustrate local packing by using rigid polymer structures and thereby increase the free volume and ion mobility. Yamamoto et al. [16] reported conductivity of $10^{-6} \mathrm{~S} / \mathrm{cm}$ at $20^{\circ} \mathrm{C}$ in rigid poly(parabanic acid) (PPA) with $T_{\mathrm{g}} \sim 350^{\circ} \mathrm{C}$ and $33 \mathrm{wt} \%$ of dissolved $\mathrm{LiCF}_{3} \mathrm{SO}_{3}$. Similarly, Wei and Shriver found high conductivities in rigid poly(1,3-dioxolan-2-one-4,5diyloxalate) (PVICOX) [17*]. Based on a slightly different idea, Ingram and coworkers achieved decoupled conductivity in side group liquid crystalline polyethers $\left[19,20^{*}, 24\right]$. It was suggested that the bulky pendent side groups in these polymers prevented the ethylene oxide backbones from adopting a close-packed structure. As a result, the conductivities of these polymers were as high as $10^{-6} \mathrm{~S} / \mathrm{cm}$ at $T_{\mathrm{g}}$. Recently, this packing frustration approach was also demonstrated by the 
Sokolov's group for a few styrene- and carbonate-based polymers [11**,13**,14*], and conductivity comparable to that of the best $\mathrm{PEO} / \mathrm{LiClO}_{4}$ was achieved.

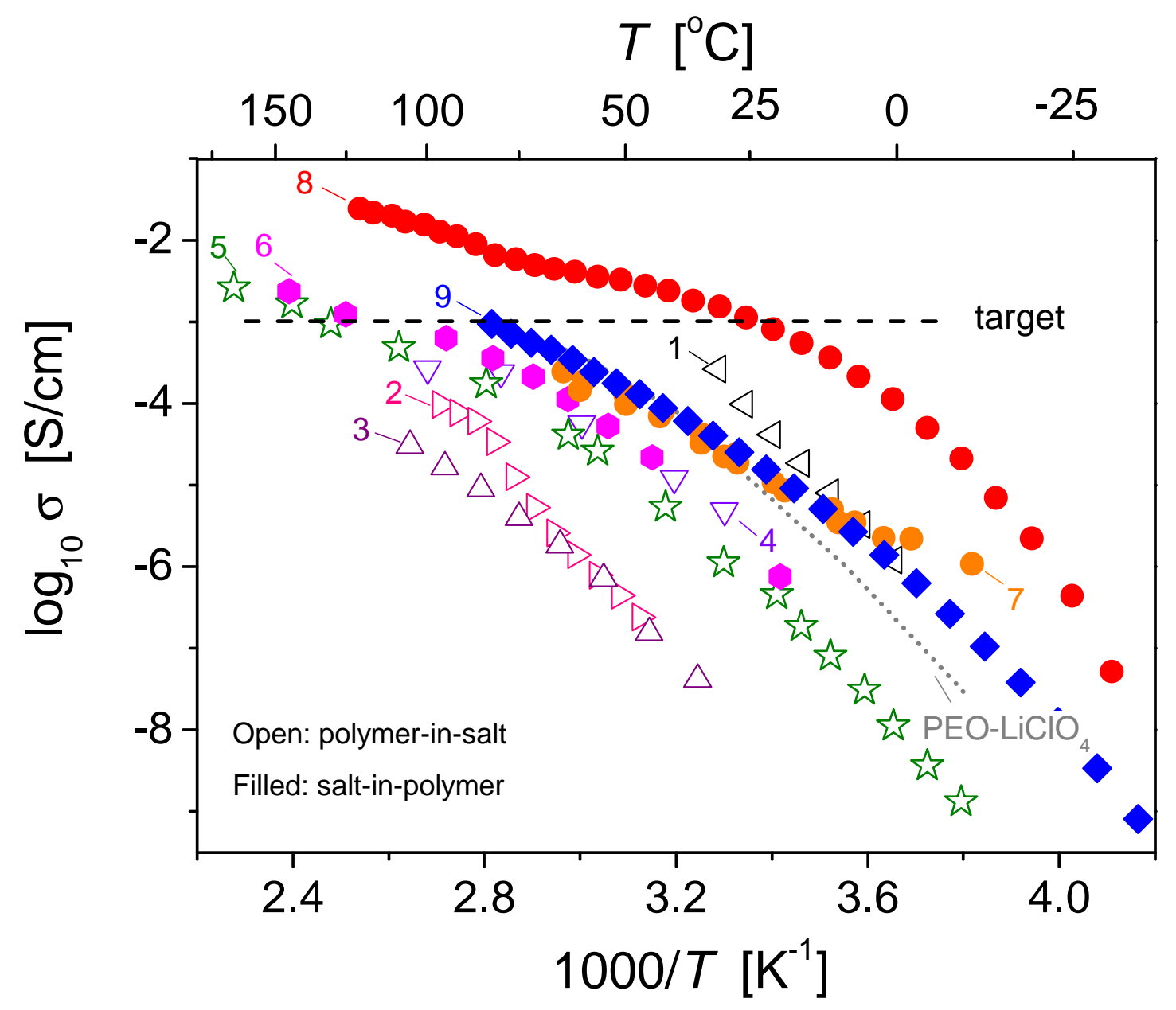

Figure 2. Temperature dependence of ionic conductivity of representative solvent-free polymer electrolytes with decoupled ion transport mechanism. The gray dotted line shows the conductivity of $\mathrm{PEO} / \mathrm{LiClO}_{4}$ (45 wt\%) [46] as a benchmark. The open symbols represent polymer-in-salt $\left[15^{*}, 17^{*}, 18,47\right]$ and the filled symbols represent salt-in-polymer [11**,16,19,20*,23]. Samples are: 1 - PVICOX $\left(\mathrm{LiCF}_{3} \mathrm{SO}_{3}, 50 \mathrm{~mol} \%\right)$ [17*], 2 - PVIC $\left(\mathrm{LiCF}_{3} \mathrm{SO}_{3}, 50 \mathrm{~mol} \%\right)$ [17*], 3 - PAN ( $\mathrm{LiCF}_{3} \mathrm{SO}_{3}, 45 \mathrm{~mol} \%$ ) [18], 4 - PEC (LiTFSI, $\left.80 \mathrm{wt} \%\right)$ [47], 5 - 90\%(50LiI-30LiOAc-20LiClO 4 )+10\%PPO [15*], 6 - PPA $\left(\mathrm{LiCF}_{3} \mathrm{SO}_{3}, 33 \mathrm{wt} \%\right)$ [16], 7 MeOC6G6 ( $\left.\mathrm{LiClO}_{4}, \mathrm{AO}: \mathrm{Li}=3: 1\right)$ [19], 8 - 0.8(0.5LiPUS-AlCl 3$)-0.2 \mathrm{LiAlCl}_{4}$ [23], and 9 - PVECPVAc (LiTFSI, $50 \mathrm{wt} \%$ ) [11**]. The horizontal dashed line indicates the target conductivity of $10^{-3} \mathrm{~S} / \mathrm{cm}$ that is required for many applications [48]. 

conductivities of several representative polymer electrolytes are shown as a function of temperature in Fig. 2, together with that of $\mathrm{PEO} / \mathrm{LiClO}_{4}$. In general, relatively high ionic conductivities have been achieved by both the polymer-in-salt and salt-in-polymer approaches. In particular, the conductivity of $0.8\left(0.5 \mathrm{LiPUS}-\mathrm{AlCl}_{3}\right)-0.2 \mathrm{LiAlCl}_{4}$, the Li-ion conducting polyimide by Zhang et al. [23], has reached the required conductivity level of $10^{-3} \mathrm{~S} / \mathrm{cm}$ at $25^{\circ} \mathrm{C}$. The polymer-in-salt electrolyte, PVICOX $\left(\mathrm{LiCF}_{3} \mathrm{SO}_{3}, 50 \mathrm{~mol} \%\right)$ [17*], also exhibits high conductivity of $\sim 10^{-4} \mathrm{~S} / \mathrm{cm}$ at $25^{\circ} \mathrm{C}$. However, it should be pointed out that some of these polymer electrolytes, including $0.8\left(0.5 \mathrm{LiPUS}-\mathrm{AlCl}_{3}\right)-0.2 \mathrm{LiAlCl}_{4}$ and $\mathrm{PVICOX}\left(\mathrm{LiCF}_{3} \mathrm{SO}_{3}, 50\right.$ mol\%), have not been thoroughly characterized. Further investigation, especially Walden plot analysis, is needed to identify the key elements leading to their apparent success. To further develop this approach, one needs to understand what controls the decoupling of ion mobility and segmental dynamics in polymers. In the early study by Sasabe and Saito [49], it was proposed that the degree of decoupling correlates with the glass transition temperature of polymers: those with high $T_{\mathrm{g}}$ have a higher degree of decoupling. The subsequent work by Wei and Shriver [17*], Ingram et al. [24] suggested that decoupling might originate from polymer packing frustration, but no quantitative analysis was given. The recent work $[12,13 * *]$ from the Sokolov's group showed that the strength of the decoupling correlates with the fragility but not with $T_{\mathrm{g}}$. Fragility, defined as $\left[\operatorname{dog}_{10} \tau / \mathrm{d}\left(T_{\mathrm{g}} / T\right)\right]_{T=T \mathrm{~g}}$, is a parameter that characterized the steepness of temperature dependence of structural relaxation time $\tau$ at $T_{\mathrm{g}}$. It has been proposed that frustration of local packing structure is responsible for both the decoupling phenomenon and the increase in fragility. Therefore, it should be possible to synthesize polymers with relatively low $T_{\mathrm{g}}$, while maintaining the decoupling properties through strong frustration in packing.

\section{Conclusions and outlook}

In this article, we have briefly reviewed the decoupling approach to solid polymer electrolytes and the new insights provided by the analysis of the relationship between ionic transport and polymer segmental relaxation.

The presented results and analysis indicate that:

$>$ There is a fundamental difference between the ionic transport mechanisms in polymers and small molecules. The ionic conductivity in polymers can be strongly decoupled from 
their segmental dynamics, in terms of both temperature dependence and relative transport rate.

$>$ Decoupling conductivity from segmental relaxation is the key for design of superionic polymers. Many polymers show the desirable characteristics similar to superionic conductors. A clear understanding of how to enhance these features is critical to the successful design of solid polymer electrolytes.

Lastly, while many polymer electrolytes should be considered as superionic in terms of the intrinsic relation between transport and structural relaxation, their sluggish segmental dynamics and mediocre ion solvation are responsible for the low ionic conductivity at ambient and low temperatures.

We hope our view could serve as a basic guideline for development of superionic polymer electrolytes. Going forward, several critical issues still need to be addressed. First, while the general connection between polymer packing frustration and decoupling has been observed, the detailed link between chemical structure and ionic transport mechanism is yet to be established. Second, the analysis of the relation between conductivity and structural relaxation has been so far limited to the case of salt-in-polymer electrolytes. As the salt concentration increases, a crossover from segmental dynamics facilitated conduction to vacancy dominated hopping conduction characteristic of solids should be expected. To obtain a complete understanding of the relation between charge transport and relaxation in polymer electrolytes, it is necessary to extend the analysis to polymer-in-salt electrolytes. Lastly, high ionic mobility must be combined with high free-ion concentration to achieve high ionic conductivity. How to identify polymer structures that promote both decoupling and ion solvation remains a challenge.

While this review is focused on the traditional polymer-salt mixtures, the decoupling approach is also critical to the design of other types of polymer electrolytes including polymeric single-ion lithium conductors and polymerized ionic liquids. They have significant advantage due to high transference number, i.e. exclusive motion of the desired ion (e.g. Li) while the counter-ion is attached to the polymer chain. In contrast to closely coupled transport mechanism in regular aprotic ionic liquids, strong decoupling of ionic conductivity from segmental relaxation has already been observed in many polymerized ionic liquids [50,51*]. A thorough investigation of the relation between ionic transport and segmental dynamics in these novel materials may provide the opportunity to further improve their properties. 


\section{Acknowledgements}

Y.Y.W. acknowledges the support by the NSF Chemistry Program (CHE-1213444). A.P.S.

acknowledges the financial support from the Division of Materials Science and Engineering, U.S. Department of Energy, Office of Basic Energy Sciences. 


\section{References and recommended reading}

1. Armand M: Polymer solid electrolytes - an overview. Solid State Ionics 1983, 9-10:745-754.

2. Ratner MA, Shriver DF: Ion Transport in Solvent-Free Polymers. Chem. Rev. 1988, 88:109-124.

3. Watanabe M, Ogata N: Ionic conductivity of polymer electrolytes and future applications. British Polym. J. 1988, 20:181-192.

4. Meyer WH: Polymer Electrolytes for Lithium-Ion Batteries. Adv. Mater. 1998, 10:439-448.

5. Wright PV: Polymer electrolytes—the early days. Electrochim. Acta 1998, 43:1137-1143.

6.** Ratner MA, Johansson P, Shriver DF: Polymer Electrolytes: Ionic Transport Mechanisms and Relaxation Coupling. MRS Bull. 2000, 25:31-37.

A comprehensive review of the ionic transport mechanism in polymer electrolytes.

7. Scrosati B, Vincent CA: Polymer Electrolytes: The Key to Lithium Polymer Batteries. MRS Bull. 2000, 25:28-30.

8.* Hallinan DT, Balsara NP: Polymer Electrolytes. Ann. Rev. Mater. Res. 2013, 43:503-525. A recent comprehensive review of polymer electrolytes.

9. Fuoss RM: Electrical Properties of Solids. VII. The System Polyvinyl Chloride-Diphenyl. J. Am. Chem. Soc. 1941, 63:378-385.

10. Baker RE: Mobility and Conductivity of Ions in and into Polymeric Solids. Pure Appl. Chem. 1976, 46:157-170.

11.** Wang Y, Fan F, Agapov AL, Saito T, Yang J, Yu X, Hong K, Mays J, Sokolov AP: Examination of the Fundamental Relation between Ionic Transport and Segmental Relaxation in Polymer Electrolytes. Polymer 2004, 55:4067-4076.

A recent analysis of the fundamental relation between ionic transport and segmental relaxation in polymer electrolytes. 
12. Agapov AL, Sokolov AP: Decoupling Ionic Conductivity from Structural Relaxation: A Way to Solid Polymer Electrolytes? Macromolecules 2011, 44:4410-4414.

13.** Wang Y, Agapov AL, Fan F, Hong K, Yu X, Mays J, Sokolov AP: Decoupling of Ionic Transport from Segmental Relaxation in Polymer Electrolytes. Phys. Rev. Lett. 2012, 108:088303.

The degree of decoupling is shown to correlate with the fragility but not with the glass transition temperature.

14.* Wang Y, Fan F, Agapov AL, Yu X, Hong K, Mays J, Sokolov AP: Design of superionic polymers-New insights from Walden plot analysis. Solid State Ionics 2014, 262:782-784.

This study demonstrates the intrinsic limitations of polyethers.

15.* Angell CA, Liu C, Sanchez E: Rubbery solid electrolytes with dominant cationic transport and high ambient conductivity. Nature 1993, 362:137-139.

The first paper on polymer-in-salt electrolytes.

16. Yamamoto T, Inami M, Kanbara T: Preparation and properties of polymer solid electrolytes using poly(vinyl alcohol) and thermally resistive poly[arylene(1,3imidazolidine-2,4,5-trione-1,3-diyl)] as matrix polymers. Chem. Mater. 1994, 6:44-50.

17.* Wei X, Shriver DF: Highly conductive polymer electrolytes containing rigid polymers. Chem. Mater. 1998, 10:2307-2308.

An early report of decoupled ionic conductivity in rigid polymer electrolytes.

18. Ferry A, Edman L, Forsyth M, MacFarlane DR, Sun J: Connectivity, ionic interactions, and migration in a fast-ion-conducting polymer-in-salt electrolyte based on poly(acrylonitrile) and $\mathrm{LiCF}_{3} \mathrm{SO}_{3}$. J. Appl. Phys. 1999, 86:2346-2348.

19. Imrie CT, Ingram MD, McHattie GS: Ion Transport in Glassy Polymer Electrolytes. $J$. Phys. Chem. B 1999, 103:4132-4138.

20.* Imrie CT, Ingram MD, McHattie GS: Ion Transport in Glassy Side-Group Liquid Crystalline Polymer Electrolytes. Advanced Materials 1999, 11:832-834. 
One of the earliest reports of decoupled ionic conductivity in side-group liquid crystalline polymer electrolytes.

21. Buriez O, Han YB, Hou J, Kerr JB, Qiao J, Sloop SE, Tian M, Wang S: Performance limitations of polymer electrolytes based on ethylene oxide polymers. J. Power Sources 2000, 89:149-155.

22. Forsyth M, Jiazeng S, MacFarlane DR: Novel high salt content polymer electrolytes based on high Tg polymers. Electrochim. Acta 2000, 45:1249-1254.

23. Zhang S, Chang Z, Xu K, Angell CA: Molecular and anionic polymer and oligomer systems with microdecoupled conductivities. Electrochimica Acta 2000, 45:1229-1236.

24. Imrie CT, Ingram MD: Decoupled ion transport in mesomorphic polymer electrolyte glasses. Electrochim. Acta 2001, 46:1413-1417.

25. Wang S-C, Tsao H-K: Ion Migration through a Polymer Solution: Microviscosity. Macromolecules 2003, 36:9128-9134.

26. Leite ER, Souza FL, Bueno PR, de Lazaro S, Longo E: Hybrid Organic-Inorganic Polymer: A New Approach for the Development of Decoupled Polymer Electrolytes. Chem. Mater. 2005, 17:4561-4563.

27. Jeong S-K, Jo Y-K, Jo N-J: Decoupled ion conduction mechanism of poly(vinyl alcohol) based Mg-conducting solid polymer electrolyte. Electrochim. Acta 2006, 52:1549-1555.

28. Souza FL, Longo E, Leite ER: Lithium Ion Motion in a Hybrid Polymer: Confirmation of a Decoupled Polyelectrolyte. ChemPhysChem 2007, 8:1778-1781.

29. Souza FL, Longo E, Leite ER: Ion Relaxation Dynamics in a Decoupled Hybrid Polyelectrolyte. ChemPhysChem 2008, 9:245-248.

30.* Ingram MD, Imrie CT: New insights from variable-temperature and variable-pressure studies into coupling and decoupling processes for ion transport in polymer electrolytes and glasses. Solid State Ionics 2011, 196:9-17. 
A recent discussion about coupling and decoupling processes for ion transport in polymer

electrolytes and glasses.

31.** Angell CA: Fast Ion Motion in Glassy and Amorphous Materials. Solid State Ionics 1983, 9-10:3-16.

An early comprehensive review of fast ion transport in amorphous materials.

32. Angell CA: Recent developments in fast ion transport in glassy and amorphous materials. Solid State Ionics 1986, 18-19, Part 1:72-88.

33. Minami T: Recent progress in superionic conducting glasses. J. Non-Cryst. Solids 1987, 95-96:107-118.

34. Adachi G-y, Imanaka N, Aono H: Fast Li $\oplus$ Conducting Ceramic Electrolytes. Adv. Mater. 1996, 8:127-135.

35. Ingram MD: Superionic glasses: theories and applications. Curr. Opin. Solid State Mater. Sci. 1997, 2:399-404.

36. Agrawal RC, Gupta RK: Superionic solid: composite electrolyte phase - an overview. $J$. Mater. Sci. 1999, 34:1131-1162.

37. Hull S: Superionics: crystal structures and conduction processes. Rep. Prog. Phys. 2004, 67:1233-1314.

38. Fan F, Wang Y, Sokolov AP: Ionic Transport, Microphase Separation, and Polymer Relaxation in Poly(propylene glycol) and Lithium Perchlorate Mixtures. Macromolecules 2013, 46:9380-9389.

39. Zwanzig R: Dielectric Friction on a Moving Ion. II. Revised Theory. J. Chem. Phys. 1970, 52:3625-3628.

40. Hubbard J, Onsager L: Dielectric dispersion and dielectric friction in electrolyte solutions. I. J. Chem. Phys. 1977, 67:4850-4857.

41. Wolynes PG: Dynamics of Electrolyte Solutions. Ann. Rev. Phys. Chem. 1980, 31:345-376. 
42. Bagchi B, Biswas R: Ionic Mobility and Ultrafast Solvation: Control of a Slow Phenomenon by Fast Dynamics. Acc. Chem. Res. 1998, 31:181-187.

43. Yoshida K, Manabe H, Takahashi Y, Furukawa T: Correlation between ionic and molecular dynamics in the liquid state of polyethylene oxide/lithium perchlorate complexes. Electrochim. Acta 2011, 57:139-146.

44. Angell CA, Ansari Y, Zhao Z: Ionic Liquids: Past, Present and Future. Faraday Discuss. 2012, 154:9-27.

45. Ferry A, Edman L, Forsyth M, MacFarlane DR, Sun J: NMR and Raman studies of a novel fast-ion-conducting polymer-in-salt electrolyte based on LiCF3SO3 and PAN. Electrochim. Acta 2000, 45:1237-1242.

46. Edman L, Ferry A, Doeff MM: Slow recrystallization in the polymer electrolyte system poly(ethylene oxide $)_{\mathbf{n}}-\mathbf{L i N}\left(\mathbf{C F}_{3} \mathbf{S O}_{2}\right)_{2}$. J. Mater. Res. 2000, 15:1950-1954.

47. Tominaga Y, Nanthana V, Tohyama D: Ionic conduction in poly(ethylene carbonate)based rubbery electrolytes including lithium salts. Polym J 2012, 44:1155-1158.

48. Wright PV: Developments in polymer electrolytes for lithium batteries. MRS Bull. 2002, 27:597-602.

49. Sasabe H, Saito S: Relationship between ionic mobility and segmental mobility in polymers in the liquid state. Polymer J. 1972, 3:624-630.

50. Nakamura K, Fukao K, Inoue T: Dielectric Relaxation and Viscoelastic Behavior of Polymerized Ionic Liquids with Various Counteranions. Macromolecules 2012, 45:38503858 .

51.* Sangoro JR, Iacob C, Agapov AL, Wang Y, Berdzinski S, Rexhausen H, Strehmel V, Friedrich C, Sokolov AP, Kremer F: Decoupling of ionic conductivity from structural dynamics in polymerized ionic liquids. Soft Matter 2014, 10:3536-3540.

A recent report of decoupled ionic conductivity in polymerized ionic liquids. 